This item was submitted to Loughborough's Research Repository by the author.

Items in Figshare are protected by copyright, with all rights reserved, unless otherwise indicated.

\title{
A cross-uncorrelator-initializer for the super-exponential algorithms in multi- user environment
}

PLEASE CITE THE PUBLISHED VERSION

PUBLISHER

(c) IEEE

VERSION

VoR (Version of Record)

\section{LICENCE}

CC BY-NC-ND 4.0

\section{REPOSITORY RECORD}

Lambotharan, Sangarapillai, Jonathon Chambers, and P. Sirisuk. 2019. "A Cross-uncorrelator-initializer for the Super-exponential Algorithms in Multi-user Environment". figshare. https://hdl.handle.net/2134/5799. 
This item was submitted to Loughborough's Institutional Repository (https://dspace.lboro.ac.uk/) by the author and is made available under the following Creative Commons Licence conditions.

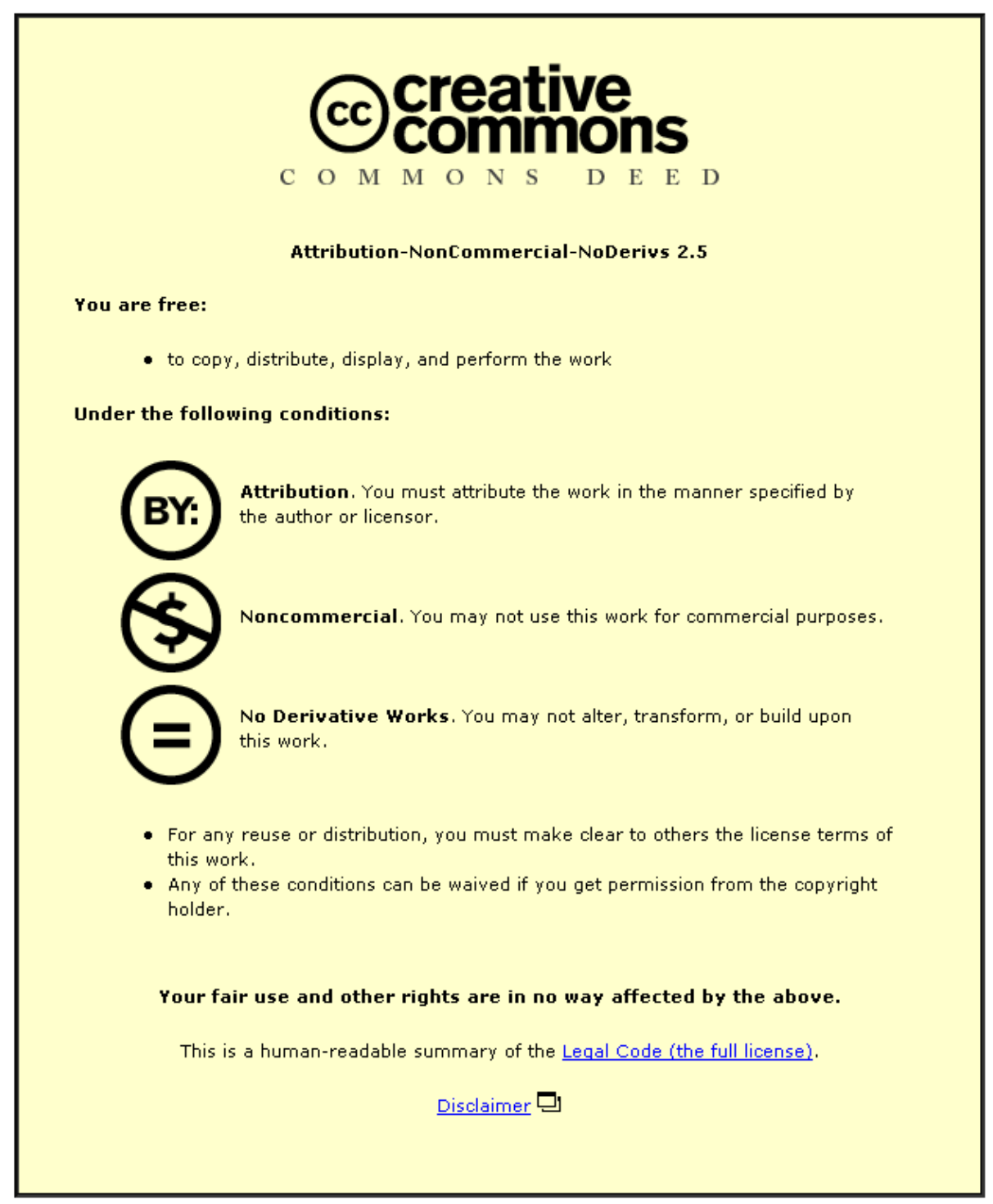

For the full text of this licence, please go to: http://creativecommons.org/licenses/by-nc-nd/2.5/ 


\title{
A CROSS-UNCORRELATOR-INITIALIZER FOR THE SUPER-EXPONENTIAL ALGORITHMS IN MULTI-USER ENVIRONMENT
}

\author{
S. Lambotharan ${ }^{1}$, J.A. Chambers ${ }^{2}$ and P. Sirisuk ${ }^{2}$ \\ ${ }^{1}$ Department of Electronics and Computer Engineering \\ Brunel University, Middlesex UB8 3PH, UK. \\ ${ }^{2}$ Department of Electrical Engineering and Electronics \\ Imperial College of Science, Technology and Medicine, London SW7 2BT, UK. \\ E-mail: j.chambers@ic.ac.uk
}

\begin{abstract}
The super-exponential algorithms (SEAs) have potential to equalize a communication channel and to mitigate interference in a multi-user environment, and possess very fast (exponential) convergence rate. By considering the basic principle behind the development of SEA, we propose a novel initialization scheme based upon the null space of a cross-correlation matrix to prevent repeated retrieval of identical sources. A sequential signal-canceler based cascaded-equalizer structure is therefore not required. Simulation results support the proposed method.
\end{abstract}

\section{INTRODUCTION .}

The super-exponential methods proposed in [1] for the blind equalization of a single-user communication channel have much attraction because they converge to a desired solution regardless of initialization, and due to their fast (exponential) convergence rate. Moreover, they do not require prior knowledge of the input distribution, except that the input has non zero Kurtosis. The SEA algorithm has been extended for a multi-user channel in [2] and [3], where SEA is required to mitigate both intersymbol interference (ISI) and interuser interference (IUI). A bank of parallel equalizers may be employed to retrieve all users, but unless there is a proper initialization scheme, more than one equalizer may retrieve identical sources. To prevent repeated retrieval of sources, a cascaded equalizer structure, in which the contribution from a previous reconstructed source is removed before the signal is sent to the next stage equalizer, has been proposed in [4] and [5]. In [5], the weight vector of the cascaded equal- izer is used to initialize a parallel equalizer structure and improvement in performance was observed. The limitation of the super-exponential method based upon a cascaded structure is that the equalizers at every stage require estimation and inversion of a new set of autocorrelation matrices. Moreover, initialization of a parallel equaliser structure from a cascaded equalizer structure requires additional computational burden. In this paper, we propose a direct initialization scheme for a structure composed of a bank of parallel equalizers for which repeated retrieval of sources is prevented. This method is based upon the null space of a crosscorrelation based matrix, but it requires only a single matrix inversion because the autocorrelation matrices for all parallel equalizers are identical.

\section{SUPER-EXPONENTIAL ALGORITHMS AND INITIALIZATION}

Consider a multi-user environment with $U$ users and $L$ sensors (antennas). There are $U L$ subchannels of order $M$. Denote the sub-channel from the $j^{\text {th }}$ source to the $i^{\text {th }}$ sensor as $c_{i j}\left(z^{-1}\right)=$ $\sum_{m=0}^{M} c_{i j}(m) z^{-m}$. For an $L$-input single-output equalizer of order $N$, the received signal vector of length $L(N+1)$ can be written as $x(k)=$ $\Delta^{H} \mathrm{~s}(k)$, where $\Delta^{H}$ is the $L(N+1) \times U(N+M+1)$ channel convolution matrix,

$$
\Delta=\left[\begin{array}{llll}
\Delta_{11}^{H} & \Delta_{12}^{H} & \cdots & \Delta_{1 U}^{H} \\
\vdots & \vdots & \vdots & \vdots \\
\Delta_{L 1}^{H} & \Delta_{L 2}^{H} & \cdots & \Delta_{L U}^{H}
\end{array}\right]^{H}
$$


$\Delta_{i j}=\left[\begin{array}{llllll}c_{i j}(0) & c_{i j}(1) & \cdots & c_{i j}(M) & \cdots & 0 \\ 0 & c_{i j}(0) & \cdots & c_{i j}(M-1) & \cdots & 0 \\ \vdots & \vdots & \vdots & \vdots & \vdots & \vdots \\ 0 & \cdots & 0 & c_{i j}(0) & \cdots & c_{i j}(M)\end{array}\right]^{H}$

The source vector $s(k)$ is of length $U(N+$ $M+1)$ and $(\cdot)^{H}$ denotes the Hermitian transpose operator. Let $\mathrm{w}_{l}$ be the weight vector of length $L(N+1)$ for the $t^{\text {th }}$ parallel equalizer. The $l^{\text {th }}$ equalizer output is written as

$$
y_{l}(k)=\mathbf{w}_{l}^{H} \mathbf{x}=\mathbf{w}_{l}^{H} \Delta^{H} \mathbf{s}(k)=\mathbf{h}_{l}^{H} \mathbf{s}
$$

where $\mathrm{h}_{l}=\Delta \mathrm{w}_{l}$ is the impulse response of the combined channel and the $l^{\text {th }}$ equalizer of length $U(N+M+1)$. Equalization and interference cancellation requires that there is only one non zero element in $\mathbf{h}_{l}$. The SEA method is aimed at performing the following two step iterative procedure, [1],

$$
\begin{gathered}
h_{l}^{[1]}(n)=\left(h_{l}(n)^{*}\right)^{p}\left(h_{l}(n)\right)^{q} \\
h_{l}^{[2]}(n)=\frac{h_{l}^{(1]}(n)}{\|\mathrm{h}\|}
\end{gathered}
$$

$n=0, \quad 1, \cdots, \quad U(N+M+1)-1, h_{l}^{(1)}(n)$ is the intermediate tap value and $h_{l}^{[2]}(n)$ is the tap value after the iteration, and $(\cdot)^{*}$ denotes complex conjugate operator. The nonnegative integers, $p$ and $q$, are such that $p+q \geq 2$. At every iteration, the largest element in $\mathrm{h}$ grows, while all other elements decay to zero. For simplicity and sufficiency, consider $p=2$ and $q=1$, which provides a solution in terms of fourth-order cumulants. The intermediate equalizer weight vector $\mathrm{w}_{l}^{\left[{ }^{1}\right]}$ must be chosen such that the distance between $\Delta w_{l}^{[1]}$ and $h_{l}^{[1]}$ is minimised, which results in the following least squares solution,

$$
\mathbf{w}_{l}^{[1]}=\left(\Delta^{H} \Delta\right)^{-1} \Delta^{H} h_{l}^{[1]}
$$

where $\Delta^{H} \Delta=\mathrm{R}$ is the autocorrelation matrix of the channel output $\mathbf{x}(k)$, and $\Delta^{H} \mathbf{h}_{l}^{[1]}=\mathrm{d}_{l}$ is an $L(N+1) \times 1$ fourth-order cumulant vector whose $n^{\text {th }}, n \in(0, L(N+1)]$, element is estimated as $\operatorname{cum}\left(y(k) y(k) y^{*}(k) x_{n}^{*}(k)\right.$, where $x_{n}(k)$ denotes the $n^{\text {th }}$ element of the channel output vector $\mathrm{x}(k)$ and

$$
\begin{array}{r}
\operatorname{cum}\left(x_{1} x_{2} x_{3} x_{4}\right)=E\left\{x_{1} x_{2} x_{3} x_{4}\right\}-E\left\{x_{1} x_{2}\right\} E\left\{x_{3} x_{4}\right\} \\
-E\left\{x_{1} x_{3}\right\} E\left\{x_{2} x_{4}\right\}-E\left\{x_{1} x_{4}\right\} E\left\{x_{2} x_{3}\right\}
\end{array}
$$

The super-exponential algorithm is the following two-step procedure which aims to provide the desired effect on $h_{l}$, (i.e., eqn. (1) and (2)), [1],

$$
\begin{gathered}
\mathrm{w}_{l}^{[1]}=\mathrm{R}^{-1} \mathrm{~d}_{l} \\
\mathrm{w}_{l}^{[2]}=\frac{\mathrm{w}_{l}^{[1]}}{\sqrt{\mathrm{w}_{l}^{[1]^{H}} \mathrm{Rw_{l }}}}
\end{gathered}
$$

$l=0,1, \cdots, U$. The ISI and IUI will be suppressed at all equalizer outputs, but SEA will not guarantee that the outputs of all the equalizers correspond to different users, i.e., more than one equalizer may retrieve the identical source.

\section{INITIALIZATION SCHEME}

The repeated retrieval of sources can however be avoided by the application of an initialization method. According to the basics of SEA, an equalizer adapted with SEA, will retrieve the $m^{\text {th }}$ source, only if the position of the largest element of $h$ (during the initialization) is between $(m-1)(M+N+1)$ and $m(M+N+1)-1$. Assume that equalizer-1 is retrieving source- $m$, therefore, equalizer- 2 should be initialized such that the position of the largest element in $h_{2}$ is not in the interval $(m-1)(M+N+1) \cdots m(M+N+1)-1$. This objective can be attained by choosing $w_{2}$ such that

$$
\sum_{\delta=-(M+N)}^{(M+N)}\left|E\left\{y_{1}(k+\delta) \mathrm{x}^{H}(k) \mathrm{w}_{2}\right\}\right|^{2}
$$

is approximately minimised. Write

$$
E\left\{y_{1}(k+\delta) \mathbf{x}^{H}(k) \mathbf{w}_{2}\right\}=\mathbf{w}_{1}^{H} \mathbf{R}_{\delta} \mathbf{w}_{2}=\mathbf{v}_{1 \delta}^{H} \mathbf{w}_{2}
$$

where $\mathrm{R}_{\delta}=E\left\{\mathrm{x}(k+\delta) \mathrm{x}^{H}(k)\right\}$ and $\mathrm{v}_{1 \delta}=\mathbf{R}_{\delta}^{H} \mathbf{w}_{1}$. Construct a $(2(M+N)+1) \times L(N+1)$ matrix $\mathrm{V}_{1}$ whose rows are

$$
\begin{aligned}
& \mathbf{v}_{1 \delta}, \delta=-(M+N), \cdots,(M+N), \text { i.e., } \\
& \mathbf{v}_{1}=\left[\begin{array}{l}
\mathbf{w}_{1}^{H} \mathbf{R}_{-(M+N)} \\
\vdots \\
\mathbf{w}_{1}^{H} \mathbf{R}_{-1} \\
\mathbf{w}_{1}^{H} \mathbf{R}_{0} \\
\mathbf{w}_{1}^{H} \mathbf{R}_{1} \\
\vdots \\
\mathbf{w}_{1}^{H} \mathbf{R}_{(M+N)}
\end{array}\right]
\end{aligned}
$$




$$
\mathrm{V}_{1}=\left[\begin{array}{l}
E\left\{y_{1}(k-(M+N)) \mathrm{x}^{H}(k)\right\} \\
\vdots \\
E\left\{y_{1}(k-1) \mathrm{x}^{H}(k)\right\} \\
E\left\{y_{1}(k) \mathrm{x}^{H}(k)\right\} \\
E\left\{y_{1}(k+1) \mathrm{x}^{H}(k)\right\} \\
\vdots \\
E\left\{y_{1}(k+(M+N)) \mathrm{x}^{H}(k)\right\}
\end{array}\right]
$$

The initial weight vector for equalizer- 2 is chosen such that the weight vector is in the nontrivial null space of $V_{1}$. This will guarantee that the second equaliser (adapted with SEA) retrieves a source that is different from the $m^{\text {th }}$ user. There can be a degree of tolerance in the accuracy of the estimation of the null space, because we do not necessarily need to make the elements of $h$ in the interval $(m-1)(M+N+1) \cdots m(M+N+1)-1$ be zero, but would like them to be small so that the position of the largest element of $h$ is not in the interval $(m-1)(M+N+1) \cdots m(M+$ $N+1)-1$.

In a similar way, the initial weight vector for the $l^{\text {th }}$ equalizer can be chosen such that the equalizer weight vector is in the nontrivial null space (denoted $\mathcal{N}_{1}$ ) of an $(l-1)$ block matrix,

$$
\left[\begin{array}{llll}
\mathrm{V}_{1}^{H} & \mathrm{~V}_{2}^{H} & \ldots & \mathrm{V}_{l-1}^{H}
\end{array}\right]^{H}
$$

A similar but not identical approach has been proposed in [6] for the global convergence of the constant modulus algorithms in the presence of noise for a single-user channel, but its application in SEA exploits the improved convergence properties of the algorithm. Notice that if $L(N+1)>$ $U(N+M+1)$, then the autocorrelation matrix $\mathrm{R}$ itself will have a nontrivial null space (denoted $\mathcal{N}_{2}$ ). In this case, the $l^{t h}$ equalizer weight vector $w_{1}$ should be chosen such that $w_{l} \in \mathcal{N}_{1}$ and $\mathrm{w}_{l} \notin \mathcal{N}_{2}$ and the ordinary inverse operator in eqn. (4) is replaced by a pseudo inverse operator. An adaptive implementation for the initializer is also possible: the initializer $\left(w_{l}\right)$ for the $l^{\text {th }}$ equalizer is obtained by adaptively minimizing the following cost function,

$$
\sum_{i=1}^{l-1} \sum_{\delta=-(M+N)}^{(M+N)}\left(\left|E\left\{y_{i}(k+\delta) x^{H}(k) w_{l}\right\}\right|^{2}\right.
$$

subject to a constraint that $E\left\{\left|y_{i}(k)\right|^{2}\right\} \neq 0$.

\section{SIMULATIONS}

A convolutive mixture of two 16-QAM sources of length 3000 is assumed. The orders of the subchannels and the equalizers are one (i.e., $M=1$ and $N=1$ ), and the number of sensors $L=3$. The impulse responses of the subchannels are

$$
\begin{aligned}
& c_{11}=\left[\begin{array}{ll}
0.3686-1.1512 i & -0.9098-0.4130 i
\end{array}\right], \\
& c_{12}=\left[\begin{array}{ll}
1.8542-2.2406 i & -0.8647-1.9676 i
\end{array}\right], \\
& c_{21}=\left[\begin{array}{ll}
-1.9198+1.1106 i & 0.5678+0.3656 i
\end{array}\right], \\
& c_{22}=\left[\begin{array}{ll}
-0.2010+0.1764 i & -0.8762-0.6835 i
\end{array}\right], \\
& c_{31}=\left[\begin{array}{ll}
1.3727-1.4072 i & -0.6288-0.2599 i
\end{array}\right], \\
& c_{32}=\left[\begin{array}{ll}
-0.5590+2.1528 i & -0.0964-1.7301 i
\end{array}\right],
\end{aligned}
$$

Equaliser-1 is adapted with SEA, and the second equalizer is initialized as proposed, and adapted with SEA. The first equalizer retrieves source-1, and the second equalizer retrieves source-2. Employing the measure of interference (intersymbol and inter-user), as [1],

$$
I(\mathrm{~h})=\frac{\sum_{n=0}^{U(M+N+1)-2}|h(n)|^{2}-\left|h_{\max }\right|^{2}}{\left|h_{\max }\right|^{2}}
$$

where $h_{\max }$ is the component of $\mathrm{h}$ having the maximal absolute value. The measure of interference as a function of iteration number and the symbol constellations for the received and recovered signal are depicted in Figure 1 and Figure 2. Figure 3 depicts the combined channel and the equalizer impulse responses for both equalizers, before and after the SEA algorithm has been performed. The initialization of the second equalizer is such that the contribution from source-1 has been eliminated (the first three elements of the impulse responses are zero), hence the second source is retrieved.

\section{CONCLUSION}

A direct initialization scheme based upon the null space of a cross-correlation matrix has been proposed for the application of super-exponential algorithms in a multi-user environment. The proposed method has potential to force all equalizer outputs to retrieve different sources for a bank of parallel equalizers. Unlike super-exponential method based upon the cascaded equalizer structure, this method does not require estimation and inversion of various autocorrelation matrices for the retrieval of different users. 


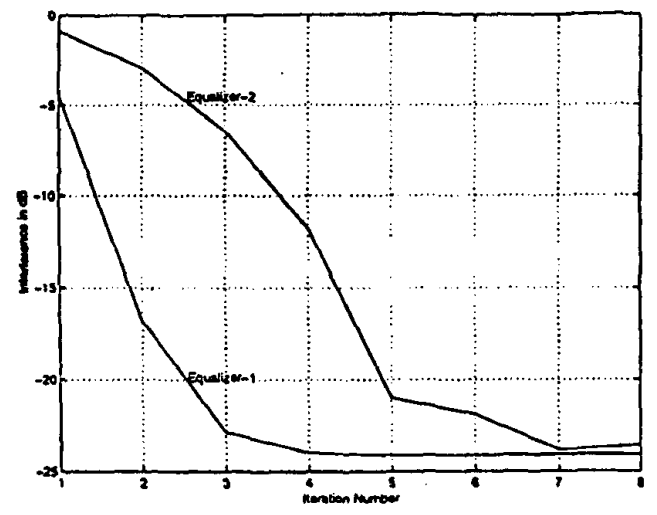

Figure 1: Measure of interference in $\mathrm{dB}$ for equalizer-1 adapted with SEA and equalizer-2 using the proposed initialization and adapted with SEA.
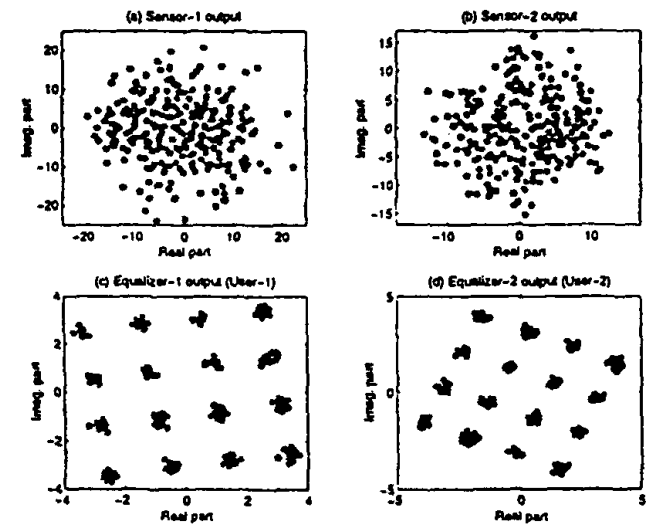

Figure 2: Sensor-1 and sensor-2 output constellations, (a) and (b), together with equalizer-1 and equalizer-2 constellations, (c) and (d), which correspond to the user- 1 and user- 2 signal.

\section{REFERENCES}

[1] O. Shalvi and E. Weinstein, "SuperExponential Methods for Blind Deconvolution," IEEE Trans. Information Theory, vol. 39, pp. 504-519, March 1993.

[2] M. Martone, "Blind Multichannel Deconvolution in Multiple Access Spread Spectrum Communications Using Higher Order Statistics," IEEE ICC, pp. 49-53, 1995.

[3] M. Martone, "Adaptive Antenna Array System for Wireless Communication Systems Using Spatial-Temporal Higher Order
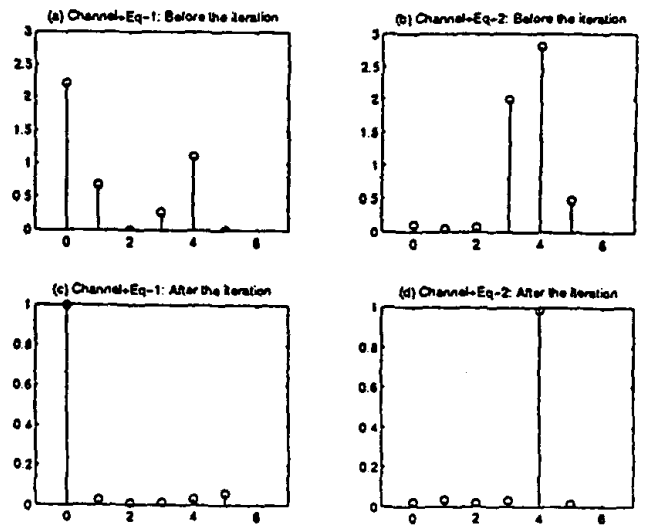

Figure 3: Combined channel and equalizer 1 and 2 impulse responses prior to the SEA iterations, (a) and (b), and after the SEA iterations, (c) and (d).

Statistics Filtering," IEEE ICC, pp. 15051508, 1995.

[4] K.L. Yeung and S.F. Yau, "A SuperExponential Algorithm for Blind Deconvolution of MIMO System," IEEE ISCAS, pp. 2517-2520, 1997.

[5] Y. Inouye and K. Tanebe, "Super Exponential Methods for Multichannel Blind Deconvolution," IEEE ICASSP, vol. 4, pp. 23532356, May 1998.

[6] S. Lambotharan, A.G. Constantinides, J.A. Chambers and $K$. Skowratananont, "A Novel Re-initialization Technique for CMA in the Presence of Channel Noise," Asilomar Conf. Sig., Sys., and Comp., Nov. 1999. 